\title{
The inhibitory effects of rosmarinic acid on catabolism induced by IL-1 $\beta$ in rat chondrocyte
}

\author{
Zhong-Nan $\mathrm{Hu}^{1}$, Li-Juan Huang ${ }^{2 \bowtie}$ and Wei-Ping Chen ${ }^{3 凶}$ \\ 'Department of Orthopedics Surgery, The Children's Hospital of Medical College, Zhejiang University, Hangzhou, People's Republic of China; \\ ${ }^{2}$ Hemodialysis Center, Division of Nursing, the Second Affiliated Hospital, School of Medicine, Zhejiang University, Hangzhou, Zhejiang Province, \\ People's Republic of China; ${ }^{3}$ Department of Orthopedics Surgery, the Second Affiliated Hospital, School of Medicine, Zhejiang University, Hang- \\ zhou, Zhejiang Province, People's Republic of China
}

The effects of rosmarinic acid (RosA) on osteoarthritis (OA) was investigated in rat chondrocytes. Chondrocytes were isolated from rat cartilage, incubated with RosA in the presence of interleukin-1beta (IL-1 $\beta)(10 \mathrm{ng} / \mathrm{ml})$. The production of IL-6, as well as the mRNA level of aggrecan (ACAN) and collagen 2 (COL2), were assessed. The gene and protein expression of $A$ disintegrin and metalloproteinase with thrombospondin motifs (ADAMTS)-4, ADAMTS-5 were also measured. RosA inhibited the production of IL- 6 as well as the gene and protein expression of ADAMTS-4 and ADAMTS-5, in addition, RosA abolished IL-1 $\beta$-induced inhibition of ACAN and COL2 gene expression. Our results suggest that RosA can inhibit extracellular matrix (ECM) degradation in OA, thus, RosA may be a possible agent in the treatment of OA.

Key words: rosmarinic acid, osteoarthritis, chondrocyte, interleukin$1 \beta$

Received: 20 April, 2018; revised: 25 September, 2018; accepted: 02 October, 2018; available on-line: 07 December, 2018

\e-mail: ze_cwp@zju.edu.cn (W-P C); 2507168@zju.edu.cn (L-J H) Abbreviations: ACAN, aggrecan; ADAMTS, A disintegrin and metalloproteinase with thrombospondin motifs; COL2, collagen 2; ECM, extracellular matrix; IL-1 $\beta$, interleukin-1 beta; IL- 6 , interleukin- 6 ; MMPs, matrix metalloproteinases; NSAIDs, nonsteroidal anti-inflammatory drugs; OA, osteoarthritis; PGEs, prostaglandins; RosA, rosmarinic acid

\section{INTRODUCTION}

Osteoarthritis (OA) is a prevalent disease in elderly people which always results in the loss of joint function (Kevorkian et al., 2004; Neogi, 2013; Glyn-Jones et al., 2015). Currently, there is lack of disease-modifying drugs for $\mathrm{OA}$ and nonsteroidal anti-inflammatory drugs (NSAIDs) are still the main agents to treat the disease (Tonge et al., 2014). Therefore, it is necessary to develop novel agents for the treatment of OA.

In normal condition, chondrocytes help to maintain the integrity of the extracellular matrix (ECM). However, during OA chondrocytes secret excessive matrix proteinases including matrix metalloproteinases (MMPs), A disintegrin and metalloproteinase with thrombospondin motifs (ADAMTS). MMPs can digest collagen 2 (COL2) and ADAMTS can digest aggrecan (ACAN) (Martel-Pelletie et al., 2001; Troeberg \& Nagase, 2012). ADAMTS-4 and ADAMTS-5 are considered important aggrecanases in the progression of OA (Verma \& Dalal, 2011; Yang et al., 2017).

Inflammatory cytokine interleukin-1-beta (IL-1 $\beta$ ) also plays a critical role in ECM degradation. IL-1 $\beta$ can in- duce increased production of MMPs and ADAMTS in OA leading to cartilage degradation.

Rosmarinic acid (RosA) is an ester of caffeic acid with many biological properties (Petersen \& Simmonds, 2003). Increasing evidence shows that Ros A exerts antiinflammatory, anti-cancer and antioxidant effects (Wu et al., 2015; Cao et al., 2016; Jin et al., 2017; Eftekhar et al., 2018). Recently, RosA was identified as a potential complementary therapeutic agent in OA treatment (Connelly, 2014). However, the effects of RosA on ADAMTS are poorly understood. In this study, we investigated the effects of RosA on ADAMTS, ACAN and COL2 in rat chondrocytes.

\section{MATERIAL AND METHODS}

Reagents. RosA, dimethyl sulfoxide (DMSO), recombinant rat IL-1 $\beta$ and MTT (3-(4,5-dimethyl-thiazole-2yl) -2,5-diphenyl tetrazolium bromide) were purchased from Sigma-Aldrich (St. Louis, MO, USA). Other reagents were purchased from Gibco BRL (Grand Island, NY, USA).

Cell culture. The study was approved by Institutional Animal Care and Use Committee of Zhejiang University (Hangzhou, China). Two-week-old Sprague-Dawley (SD) rats were used as the source for chondrocytes culture. The cartilage from the knee joints of the rats was digested with $0.2 \%$ collagenase II and the chondrocytes were isolated as previously described (Chen et al., 2012). Then, the chondrocytes were cultured in DMEM supplemented with $10 \%$ FBS, $100 \mathrm{U} / \mathrm{ml}$ penicillin and $100 \mathrm{U} / \mathrm{ml}$ streptomycin. The experiments were conducted during cell passages 3 to 4 .

Cell viability. The chondrocytes were seeded in the 96-well plates $\left(5 \times 10^{3} /\right.$ well $)$ with RosA at different concentrations for $24 \mathrm{~h}$ and $48 \mathrm{~h}$, the culture medium was removed and MTT solution $(5 \mathrm{mg} / \mathrm{ml})$ was added (20 $\mu \mathrm{l} /$ well). Then, the solution was removed and DMSO (150 $\mu \mathrm{l} /$ well) was added. The optical density (OD) at $570 \mathrm{~nm}$ was determined using a microplate reader (BioRad, Hercules, CA, USA).

Measurement of IL-6 production. Chondrocytes were seeded in six-well plates $\left(1 \times 10^{5} /\right.$ well $)$, cells were serum starved overnight and incubated with different concentrations of RosA for $1 \mathrm{~h}$ in the presence of IL-1 $\beta$ (10 $\mathrm{ng} / \mathrm{mL})$ for $24 \mathrm{~h}$. The culture medium was collected and subjected to IL- 6 production assay by ELISA kit (R\&D Systems, Minneapolis, MN, USA). The chondrocytes were collected for gene expression analysis.

Quantitative real-time polymerase chain reaction (PCR). Total RNA from the chondrocytes 


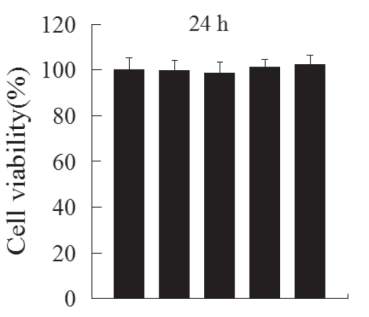

$\operatorname{Ros} A(\mu \mathrm{M}) \quad 0 \quad 10 \quad 25 \quad 50 \quad 100$

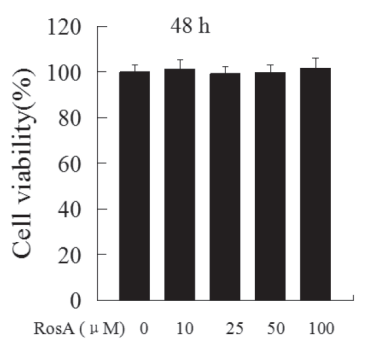

Figure 1. Effect of RosA on chondrocytes viability.

Cells were cultured in 96-well plates and incubated with RosA $(0-100 \mu \mathrm{M})$ for $24 \mathrm{~h}$ and $48 \mathrm{~h}$. Cell viability was determined using the MTT assay. The experiment was performed in three replicates.

was isolated using TRIzol reagent according to the manufacturer's protocol (Invitrogen, Carlsbad, CA, USA) and reversed-transcribed using the Moloney murine leukemia virus reverse transcriptase cDNA synthesis kit (Promega, Madison, WI, USA). cDNA samples were amplified and quantified by qPCR using the iCycler apparatus system (Bio-Rad). The primers for amplification of each gene were as follows: for ADAMTS-4: 5'-CATCCTACGCCGGAAGAGTC-3' and 5'-AAGCGAAGCGCT'TGT'T'TCTG-3'; for ADAMTS-5: 5'-CCCAAATACGCAGGTGTCCT-3' and 5'-ACACACGGAGTTGCTGTAGG-3'; for COL2: 5'-TCAAGTCGCTGAACAACCAG-3' and 5'-GTCTCCGCTCT'TCCACTCTG-3'; for ACAN: 5'-GCAGCACAGACACT'TCAGGA-3' and 5'-CCCAC'T'TCTACAGGCAAGC-3'; for GAPDH: 5'-TGTGAACGGAT'T'TGGCCGTA-3' and 5'-TGAACT'TGCCGTGGGTAGAG-3'. GAPDH was used as an internal control. The real-time PCR data were quantified using the $2^{-\Delta \Delta \mathrm{CT}}$ method.

Western blotting analysis. The protein was extracted from the chondrocytes, resolved by sodium dodecyl sulfate-polyacrylamide gel electrophoresis (SDS-PAGE) and transferred to polyvinyl fluoride (PVDF) membranes. The membranes were incubated with primary antibodies (ADAMTS-4/5, COL2 and $\beta$-actin) (Cell Signaling Technology, Beverly, MA, USA). After washing the membranes were incubated for $1 \mathrm{~h}$ at room temperature with HAP-conjugated secondary antibodies. Finally, the membranes were incubated with the electroencephalographic substrate and exposed to X-ray film (Kodak). All of the assays were performed in triplicate.

Statistical analysis. Data are expressed as the mean \pm standard deviation (SD) and were analyzed statistically using one-way ANOVA. The Dunnett-t method was used as the post-test in the ANOVA. Differences were considered significant when $p$ values were lower than 0.05. Data analysis was performed using SPSS 15.0

\section{RESULTS}

\section{Effect of RosA on cell viability}

First, we assessed the effect of RosA on cell viability using MT'T assay. RosA at a concentration of $100 \mu \mathrm{M}$ did not show toxicity to the chondrocytes (Fig. 1).

\section{RosA decreased the production of IL- 6}

The production of IL- 6 in the culture media was investigated by ELISA. IL-1 $\beta$ stimulation resulted in

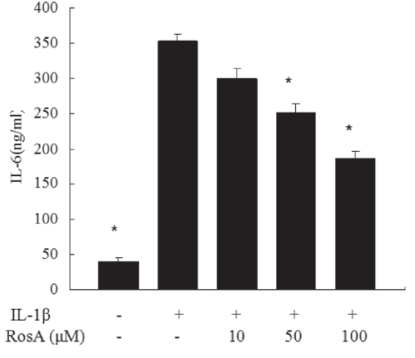

Figure 2 Effect of RosA on IL-6 production.

Cells were cultured in the absence or in the presence of IL-1 $\beta(10 \mathrm{ng} / \mathrm{ml})$ and with or without RosA. Conditioned media was collected for IL-6 measurement using ELISA kit. Values are presented as means and SDs. ${ }^{*} p<0.05$ compared to cells stimulated with IL-1 $\beta$ alone. The experiment was performed in three replicates.
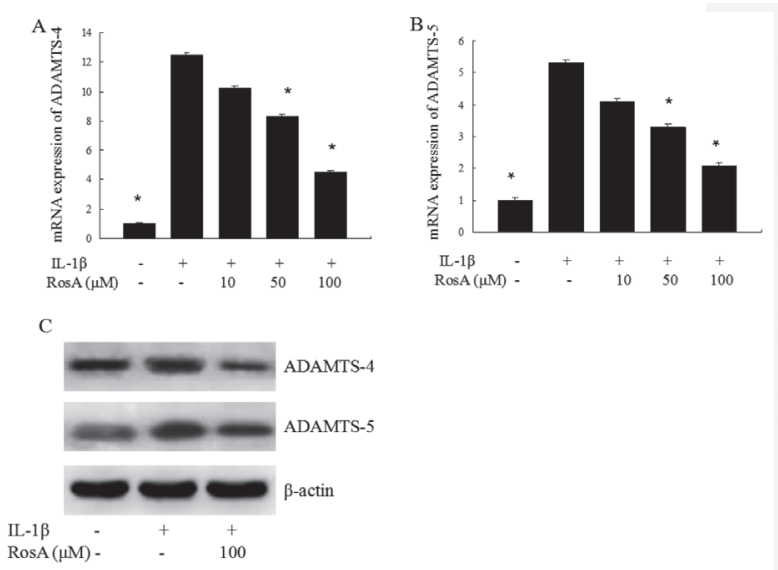

Figure 3. Effects of RosA on gene and protein expressions of ADAMTS-4 and ADAMTS-5 in IL-1 $\beta$-induced rat chondrocytes.

Cells were cultured in the absence or in the presence of IL-1 $\beta$ (10 $\mathrm{ng} / \mathrm{ml}$ ) and with or without RosA. mRNA and protein level were detected by real-time quantitative PCR and Western blotting, respectively. Values are presented as means and SDs. ${ }^{*} p<0.05 \mathrm{com}$ pared to cells stimulated with IL-1 $\beta$ alone. The experiment was performed in three replicates.

up-regulation of the production of IL-6, whereas RosA at the concentration of 50 and $100 \mu \mathrm{M}$ inhibited the production of IL-6 (Fig. 2).

\section{RosA inhibited the IL- $1 \beta$-induced expression of ADAMTS-4 and ADAMTS-5}

In order to investigate the effects of RosA on ADAMTS in the presence of IL-1 $\beta$, the gene and protein expression of ADAMTS- 4 and ADAMTS-5 in the chondrocytes were measured. IL- $1 \beta$ stimulation significantly increased the expression of ADAMTS-4 and ADAMTS-5 in the chondrocytes. RosA at the concentration of 50 and $100 \mu \mathrm{M}$ significantly reduced ADAMTS-4 and ADAMTS- 5 expression in IL- $1 \beta$-induced chondrocytes $(p<0.05)$ (Fig. 3).

RosA increased the gene expression of ACAN and COL2 in chondrocytes

IL-1 $\beta$ significantly decreased the mRNA level of ACAN and COL2. RosA at the concentration of 50 and $100 \mu \mathrm{M}$ in combination with IL-1 $\beta$ significantly increased ACAN and COL2 gene expression in chondrocytes $(p<0.05)$ (Fig. 4). 


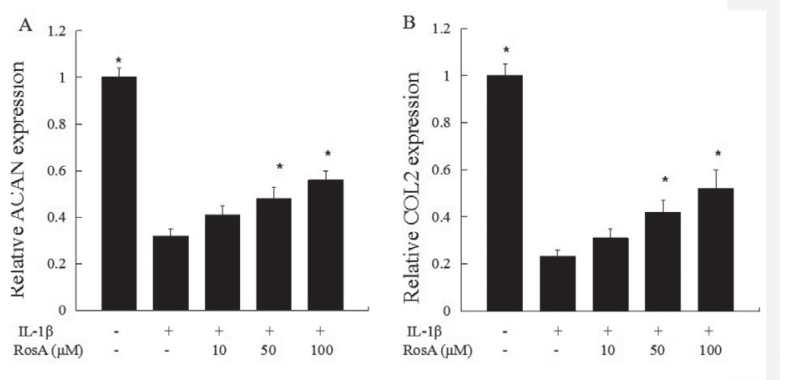

Figure 4. Effects of RosA on ACAN and COL2 gene expression in IL-1 $\beta$ induced chondrocytes.

Cells were cultured in the absence or in the presence of IL-1 $\beta$ $(10 \mathrm{ng} / \mathrm{ml})$ and with or without RosA. mRNA level was measured by real-time quantitative PCR. Values are presented as means and SDs. * $p<0.05$ compared with cells stimulated with IL-1 $\beta$ alone. The experiment was performed in three replicates.

\section{DISCUSSION}

In this study, we assessed the effects of RosA on the expression of anabolic and catabolic mediators. Our results showed that RosA can antagonize the catabolic effects of IL-1 $\beta$ in chondrocytes.

$\mathrm{OA}$ is a multifactorial disease mainly characterised by articular cartilage destruction. It is well-known that COL2 and ACAN are the main components of the cartilage ECM. In the normal cartilage, there is a balance between ECM anabolism and catabolism. However, in $\mathrm{OA}$ the balance is disturbed in favor of the catabolism. Depletion of AGAN and COL2 leads to cartilage degradation in OA (Mankin \& Lippiello, 1970). Previous studies showed that ADAMTS-4 and ADAMTS-5 are responsible for ACAN depletion in osteoarthritic cartilage (Gendron et al., 2007). It is well-known that these two aggrecanases cleave the aggrecan core protein at the aggrecanase-specific Glu373-Ala374 bond in the aggrecan interglobular domain (IGD) region (Abbaszade et al., 1999; Tortorella et al., 2001). Thus, ADAMTS-4 and ADAMTS-5 received much attention in research aiming to find a new potential agent for OA treatment (Apte, 2016; Verma et al., 2016). In the present study, we also investigated the effects of RosA on ADAMTS-4 and ADAMTS-5 expression, and our results showed that RosA can inhibit the gene and protein expression of ADAMTS-4 and ADAMTS-5 in chondrocytes. In addition, we examined the effects of RosA on the expression of ACAN and COL2, and we found that RosA can up-regulate the gene expression of ACAN and COL2. Together, these results suggest that RosA treatment can inhibit the degradation of cartilage ECM, leading to the suppression of articular cartilage degradation.

It is established that $\mathrm{OA}$ is also an inflammatory disease and that inflammation plays an important role in the progression of OA (Berenbaum, 2013). Previous studies showed that several pro-inflammatory cytokines, chemokines and prostaglandins (PGEs) were involved in OA (Robinson et al., 2016; Scanzello, 2017). These inflammatory mediators induced the inflammatory reaction in chondrocytes, leading to synovial inflammation and articular cartilage degradation. As one of the most important inflammatory factors in OA, IL-1 $\beta$ is involved in synovial inflammation, apoptosis of chondrocytes and ECM catabolism, furthermore, IL-1 $\beta$ could induce the expression of aggrecanases in OA (Koshy et al., 2002; Daheshia \& Yao, 2008). In the present study, we treated the chondrocytes with IL-1 $\beta$, as expected, the expression of ADAMTS-4 and ADAMTS-5 was increased after incubation with IL-1 $\beta$, and the production of IL6 , an inflammatory cytokine, was also upregulated by IL-1ß. RosA is known to exhibit anti-inflammatory activities, Rui and others (Rui et al., 2017) reported that RosA inhibited the expression of IL-6 in 3T3-L1 adipocytes. Our results showed that RosA can inhibit the secretion of IL-6.Therefore, the protective effects of RosA on chondrocytes may be partly associated with its antiinflammatory properties. However, Eo and others (Eo et al., 2017) reported that RosA can induce COX-2, another inflammatory cytokine, in rabbit chondrocytes. Thus, the role of RosA in OA is still unclear.

In conclusion, we found that RosA inhibited IL-1 $\beta$ upregulated expression of ADAMTS-4, ADAMTS-5 and IL-6. We speculate that RosA exerts its role in OA via inhibiting matrix-degrading enzymes and inflammatory factors. Our results indicate that RosA may possess chondroprotective effect in OA.

\section{Competing Interests}

The author(s) declare that they have no competing interests.

\section{Acknowledgements}

This study was supported by the National Natural Science Foundation of China (81371996) and Health and Family Planning Commission of Zhejiang Province (2016KYA098).

\section{REFERENCES}

Abbaszade I, Liu RQ, Yang F, Rosenfeld SA, Ross OH, Link JR, Ellis DM,Tortorella MD, Pratta MA, Hollis JM, Wynn R, Duke JL, George HJ, Hillman Jr KM, Wiswall BH, Copeland RA, Decicco CP, Bruckner R, Nagase H, Itoh Y, Newton RC, Magolda RL, Trzaskos JM, Hollis GF, Arner EC, Burn TC (1999) Cloning and characterization of ADAMTS11, an aggrecanase from the ADAMTS family. J Biol Chem 274: 23443-23450. doi: 10.1074/jbc.274.33.23443

Apte SS (2016) Anti-ADAMTS5 monoclonal antibodies: implications for aggrecanase inhibition in osteoarthritis. Biochem J 473: e1-e4. doi: 10.1042/BJ20151072

Berenbaum F (2013) Osteoarthritis as an inflammatory disease (osteoarthritis is not osteoarthrosis!). Osteoarthritis Cartilage 21: 16-21. doi: 10.1016/j.joca.2012.11.012

Cao W, Hu C, Wu L, Xu L, Jiang W (2016) Rosmarinic acid inhibits inflammation and angiogenesis of hepatocellular carcinoma by suppression of NF- $x \mathrm{~B}$ signaling in $\mathrm{H} 22$ tumor-bearing mice. J Pharmacol Sci 132: 131-137. https://doi.org/10.1016/j.jphs.2016.09.003

Chen WP, Hu PF, Bao JP, Wu LD (2012) Morin exerts antiosteoarthritic properties: an in vitro and in vivo study. Exp Biol Med 237: 380-386. doi: 10.1258/ebm.2011.011271

Connelly AE, Tucker AJ, Tulk H, Catapang M, Chapman L, Sheikh N, Yurchenko S, Fletcher R, Kott LS, Duncan AM, Wright AJ (2014) High-rosmarinic acid spearmint tea in the management of knee osteoarthritis symptoms. J Med Food 17: 1361-1367. https:// doi.org/10.1089/jmf.2013.0189

Daheshia M, Yao JQ (2008) The interleukin 1beta pathway in the pathogenesis of osteoarthritis. J Rheumatol 35: 2306-23012. https:// doi.org/10.3899/jrheum.080346

Eftekhar N, Moghimi A, Boskabady MH (2018) Prophylactic effect of rosmarinic acid on tracheal responsiveness, white blood cell count and oxidative stress markers in lung lavage of sensitized rats. Pharmacol Rep 70: 119-125. https://doi.org/10.1016/j.pharep.2017.08.010

Eo SH, Kim SJ (2017) Rosmarinic acid induces rabbit articular chondrocyte differentiation by decreases matrix metalloproteinase-13 and inflammation by upregulating cyclooxygenase- 2 expression. $J$ Biomed Sci 24: 75. doi: 10.1186/s12929-017-0381-5

Gendron C, Kashiwagi M, Lim NH, Enghild JJ, Thøgersen IB, Hughes C, Caterson B, Nagase H (2007) Proteolytic activities of human ADAMTS-5: Comparative studies with ADAMTS-4. J Biol Chem 282: 18294-18306. doi: 10.1074/jbc.M701523200

Glyn-Jones S, Palmer AJ, Agricola R, Price AJ, Vincent TL, Weinans H, Carr AJ (2015) Osteoarthritis. Lancet 386: 376-387. https://doi. org/10.1016/S0140-6736(14)60802-3

Jin BR, Chung KS, Cheon SY, Lee M, Hwang S, Noh Hwang S, Rhee $\mathrm{KJ}$, An HJ (2017) Rosmarinic acid suppresses colonic inflammation 
in dextran sulphate sodium (DSS)-induced mice via dual inhibition of NF- $x \mathrm{~B}$ and STAT3 activation. Sci Rep 7: 46252.

Kevorkian L, Young DA, Darrah C, Donell ST, Shepstone L, Porter S, Brockbank SMV, Edwards DR, Parker AE, Clark IM (2004) Expression profiling of metalloproteinases and their inhibitors in cartilage. Arthritis Rheum 50: 131-141. https://doi.org/10.1002/art.11433

Koshy PJT, Lundy CJ, Rowan AD, Porter S, Edwards DR, Hogan A, Clark IM, Cawston TE (2002) The modulation of matrix metalloproteinase and ADAM gene expression in human chondrocytes by interleukin-1 and oncostatin M: A time-course study using real-time quantitative reverse transcriptionpolymerase chain reaction. Arthritis Rheum 46: 961-967. https://doi.org/10.1002/art.10212

Martel-Pelletier J, Welsch DJ, Pelletier JP (2001) Metalloproteases and inhibitors in arthritic diseases. Best Pract Res Clin Rheumatol 15: 805829. https://doi.org/10.1053/berh.2001.0195

Mankin HJ, Lippiello L (1970) Biochemical and metabolic abnormalities in articular cartilage from osteo-arthritic human hips. J Bone Joint Surg Am 52: 424-434

Neogi T (2013) The epidemiology and impact of pain in osteoarthritis. Osteoarthritis Cartilage 21: 1145-1153. https://doi.org/10.1016/j. joca.2013.03.018

Petersen M, Simmonds MS (2003) Rosmarinic acid. Phytochemistry 62: 121-125

Robinson WH, Lepus CM, Wang Q, Raghu H, Mao R, Lindstrom TM, Sokolove J (2016) Low-grade inflammation as a key mediator of the pathogenesis of osteoarthritis. Nat Rev Rheumatol 12: 580-592. doi: 10.1038 /nrrheum.2016.136

Rui Y, Tong L, Cheng J, Wang G, Qin L, Wan Z (2017) Rosmarinic acid suppresses adipogenesis, lipolysis in 3T3-L1 adipocytes, lipopolysaccharide-stimulated tumor necrosis factor- $\alpha$ secretion in macrophages, and inflammatory mediators in 3T3-L1 adipocytes. Food Nutr Res 61: 1330096. doi: 10.1080/16546628.2017.1330096
Scanzello CR (2017) Chemokines and inflammation in osteoarthritis: Insights from patients and animal models. J Orthop Res 35: 735-739. doi: 10.1002/jor.23471

Tonge DP, Pearson MJ, Jones SW (2014) The hallmarks of osteoarthritis and the potential to develop personalised diseasemodifying pharmacological therapeutics. Osteoarthritis Cartilage 22: 609-621. https://doi.org/10.1016/j.joca.2014.03.004

Tortorella MD, Malfait AM, Deccico C, Arner E (2001) The role of ADAM-TS4 (aggrecanase-1) and ADAM-TS5 (aggrecanase-2) in a model of cartilage degradation. Osteoarthritis Cartilage 9: 539-552. https://doi.org/10.1053/joca.2001.0427

Troeberg L, Nagase H (2012) Proteases involved in cartilage matrix degradation in osteoarthritis. Biochim Biophys Acta 1824: 133-145. https://doi.org/10.1016/j.bbapap.2011.06.020

Verma P, Dalal K (2011) ADAMTS-4 and ADAM TS-5: Key Enzymes in Osteoarthritis. J Cell Biochem 112: 3507-3514. https://doi. org/10.1002/jcb.23298

Verma P, Dalal K, Chopra M (2016) Pharmacophore development and screening for discovery of potential inhibitors of ADAMTS-4 for osteoarthritis therapy. J Mol Model 22: 178. doi: 10.1007/s00894016-3035-8

Wu CF, Hong C, Klauck SM, Lin YL, Efferth T (2015) Molecular mechanisms of rosmarinic acid from Salvia miltiorrbiza in acute lymphoblastic leukemia cells. J Ethnopharmacol 176: 55-68. https:// doi.org/10.1016/j.jep.2015.10.020

Yang CY, Chanalaris A, Troeberg L (2017) ADAMTS and ADAM metalloproteinases in osteoarthritis-looking beyond the 'usual suspects'. Osteoarthritis Cartilage 25: 1000-1009. https://doi.org/10.1016/j. joca.2017.02.791 